Proceedings of the 42th "Jaszowiec" International School and Conference on the Physics of Semiconductors, Wisła 2013

\title{
Quantum Effects in Electrical Conductivity and Photoconductivity of Single SbSI Nanowire
}

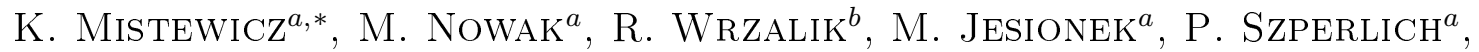 \\ R. PASZKIEWICZ ${ }^{c}$ AND A. GUISEPPI-EliE ${ }^{d}$ \\ ${ }^{a}$ Institute of Physics, Silesian University of Technology, Z. Krasińskiego 8, 40-019 Katowice, Poland \\ ${ }^{b}$ Institute of Physics, University of Silesia, Bankowa 14, 40-007 Katowice, Poland \\ ${ }^{c}$ Division of Microelectronics and Nanotechnology, Wrocław University of Technology \\ Długa 65, 53-633 Wrocław, Poland \\ ${ }^{d}$ Center for Bioelectronics, Biosensors and Biochips, Clemson University, 132 Earle Hall, Clemson, USA \\ For the first time current quantization is reported for antimony sulfoiodide (SbSI) nanowires. It has been \\ registered in current responses on electric field switching as well as on illumination on and off. Current steps \\ determined in all experiments have been equal to each other within the experimental error. It has been explained \\ by the quantized change of free carrier concentration in nanowire. Lateral dimensions of SbSI nanowires estimated \\ from quantum steps are comparable with geometrical data reported for the same technology of material preparation.
}

DOI: 10.12693 /APhysPolA.124.827

PACS: 81.07.Vb, 81.07.Bc, 73.63.Bd

\section{Introduction}

All around the world a great deal of attention is focused on one-dimensional nanostructures because of their properties related to quantum effects, which may be promising for various electronics and optoelectronics applications. To the best of our knowledge, the quantum effects in antimony sulfoiodide (SbSI) nanowires have never been reported before. Simultaneously, the SbSI (being a semiconducting ferroelectric [1]) is a promising material due to a large number of interesting properties, e.g., pyroelectric, pyrooptic, piezoelectric, and electrooptic. Measurements of current quantization in current responses on the switch on of voltages and illumination of SbSI nanowires have been the aim of this paper.

\section{Experiment}

The route of preparing single SbSI nanowires on $\mathrm{Si} / \mathrm{SiO}_{2}$ substrates as well as glass substrates (model IAME-co-IME2-1AU made by Abtech Scientific Inc.) equipped with interdigitated gold electrodes (with $l=$ $1 \mu \mathrm{m}$ spacing) can be summarized as follows. First of all, SbSI gel was prepared sonically from the constituents (the elements $\mathrm{Sb}, \mathrm{S}$ and I) in ethanol. This gel consisted of large number of crystalline nanowires with average lateral dimensions of $10-50 \mathrm{~nm}$ and average lengths up to several micrometers. The used experimental setup, the applied procedure, and characterization of the product were the same as described in [2]. After this ethanol was replaced with toluene and the product (in ratio: $0.7 \mathrm{mg}$ $\mathrm{SbSI}$ gel/1 ml toluene) was again sonicated to disperse

*corresponding author; e-mail: krystian.mistewicz@polsl.pl the solidified gel. As prepared SbSI sol was dropped upon substrates in very small volume using insulin syringe equipped with $31 \mathrm{G}$ needle. In the case of IAME-co-IME2-1AU substrates, dc electric field of $5 \times 10^{5} \mathrm{~V} / \mathrm{m}$ was applied between electrodes during the deposition of SbSI sol to align the nanowires perpendicularly to the electrodes. Then the sample was dried in a glove box, in room temperature, in order to evaporate the toluene. In next stage SbSI nanowires deposited on the $\mathrm{Si} / \mathrm{SiO}_{2}$ substrate were ultrasonically welded to the metal electrodes applying technique similar to the reported in [3]. We used ultrasonic generator ADG70-100P-230-NO (Rinco Ultrasonics) with working frequency $70 \mathrm{kHz}$ and max power $120 \mathrm{~W}$, transducer C 70-2 (Rinco Ultrasonics) and a special sonotrode made by ourselves with $\mathrm{SiC}$ single crystal end. Ultrasonic nanowelding was carried out by applying one ultrasonic impulse of duration $90 \mathrm{~ms}$ and amplitude $0.05 \mu \mathrm{m}$. Press force of the welding head on the sample was equal to $0.96 \mathrm{~N}$. Energy realized during the welding process amounted to $2.3 \mathrm{~J}$.

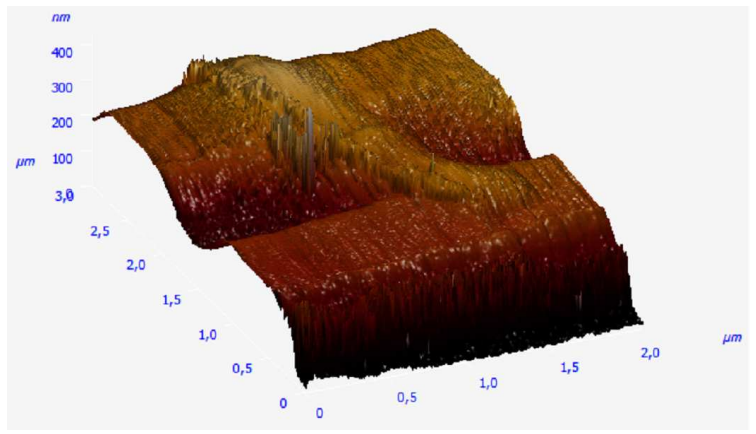

Fig. 1. AFM image of a single SbSI nanowire ultrasonically welded to interdigitated electrodes on the $\mathrm{Si} / \mathrm{SiO}_{2}$ substrate. 
Figure 1 presents a single SbSI nanowire ultrasonically welded to interdigitated electrodes on the $\mathrm{Si} / \mathrm{SiO}_{2}$ substrate. The atomic force microscopy (AFM) investigations were carried out using atomic force microscope Solver P47H-PRO (NT-MDT).

The electrical connections between gold electrodes and the measurement board were made using HB05 thermosonic wire bonder (TPT Wire Bonder). All electric measurements were done using the Keithley 6517A electrometer in normal atmosphere at $298 \mathrm{~K}$ temperature. Acquisition of the data and control of the applied voltage were realized using a PC computer with IEEE 488 bus and appropriate programs in LabView 7.5 (National Instruments).

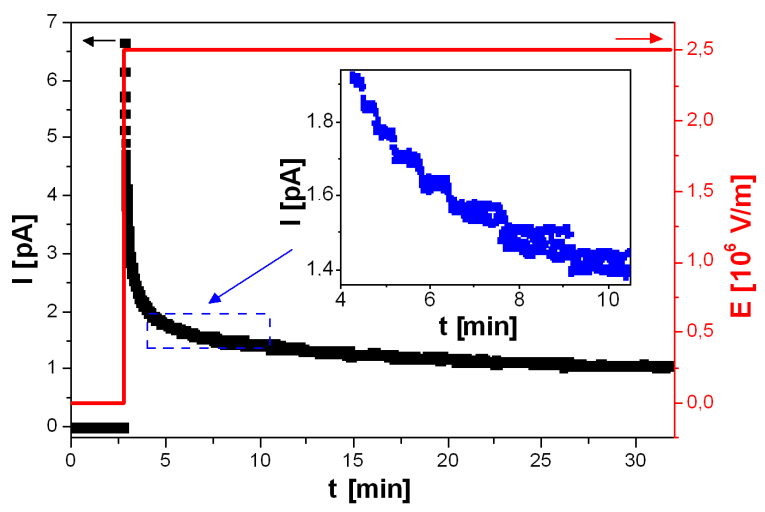

Fig. 2. Current response (-) on electric field (solid curve) applied to SbSI single nanowire. Inset in figure shows the magnification of area indicated by dashed rectangle.

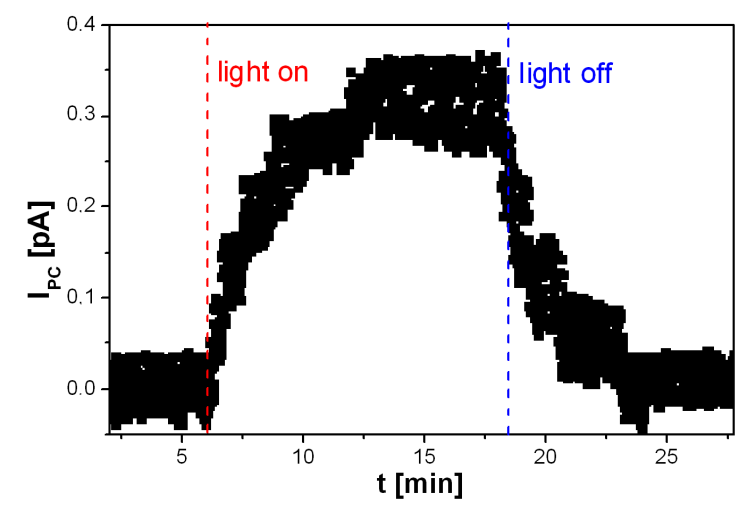

Fig. 3. Kinetics of photoconductivity current for single SbSI nanowire. Dashed vertical lines indicate the illumination switch on and switch off.

Figure 2 presents current response on electric field applied to the unilluminated SbSI single nanowire. After quick response the current decreases in step-like form (see inset in Fig. 2). Magnitudes of the 32 observed current steps are practically the same. The averaged value of the current step was $\Delta I_{\mathrm{D}}=66(4) \mathrm{fA}$.
Figure 3 shows kinetics of photoconductivity current for a single SbSI nanowire illuminated with visible light. Intensity of the bias electric field was $2.5 \times 10^{6} \mathrm{~V} / \mathrm{m}$. The observed photoconductivity current steps had the average value $\Delta I_{\mathrm{PC}}=60(7) \mathrm{fA}$.

\section{Discussion}

The quick increase followed by a slow decrease of a current after electric field switch on is characteristic for the trapping of carriers at boarders of electric domains in SbSI [1]. Authors of [4] reported that SbSI in paraelectric phase (i.e. in $T=298 \mathrm{~K}$ ) consists of irregular positively or negatively polarized nanodomains. The nanodomains about $0.5 \mathrm{~nm}$ thick, $2.0 \mathrm{~nm}$ broad and a few $\mathrm{nm}$ long are elongated in the [001] direction, i.e. along SbSI nanowire. Therefore the observed quantization effect (Fig. 2) can be explained as a result of the change of free carrier concentration with time after polarization of the nanodomains. Also in the case of illumination of SbSI nanowire the change of free carrier concentration with time (Fig. 3) can explain the observed quantization of photoconductivity current. It should be noticed that the current steps $\Delta I_{\mathrm{D}}$ and $\Delta I_{\mathrm{PC}}$ are equal to each other within the experimental error. It seems to be a proof of the same nature of quantization effects observed in electrical conductivity (Fig. 2) and photoconductivity (Fig. 3).

Let us assume that the observed changes of electrical conductivity and photoconductivity in SbSI nanowire are represented by the elementary unit of conductance

$$
\Delta \sigma=e^{2} / h,
$$

where $e$ is electron charge, $h$ is Planck's constant. Then, applying the determined value of current step into simple formula describing electrical conductivity of a nanowire with square cross-section, one can estimate the nanowire lateral dimension

$$
d=\frac{1}{e} \sqrt{\frac{h \Delta I_{\mathrm{A}}}{E}},
$$

where $\Delta I_{\mathrm{A}}=\Delta I_{\mathrm{D}}$ for the measurements on electrical conductivity in darkness or $\Delta I_{\mathrm{A}}=\Delta I_{\mathrm{PC}}$ for the measurements on photoconductivity. Hence, lateral dimension of SbSI nanowire determined from the time dependence of dark current (Fig. 2) and photoconductivity current (Fig. 3) have been estimated as 26.1(8) $\mathrm{nm}$ and $25(2) \mathrm{nm}$, respectively. These values are very comparable to the lateral dimensions of SbSI nanowires fabricated with the applied sonochemical technique and reported in $[2,5,6]$.

\section{Conclusions}

For the first time the quantum effects in conductivity and photoconductivity of single SbSI nanowires have been observed. Lateral dimensions of SbSI nanowires calculated from quantum steps in two different experiments (electrical conductivity and photoconductivity measurements) are very close to each other. In addition, they are comparable with values of lateral dimensions of SbSI 
nanowires reported in literature for the same technology of material preparation.

\section{Acknowledgments}

This paper was partially supported by the National Science Centre (Poland) under contract No. 2011/01/B/ ST5/06273.

\section{References}

[1] V.M. Fridkin, Ferroelectric Semiconductors, Consultants Bureau, New York 1980
[2] M. Nowak, Ł. Bober, B. Borkowski, M. Kępińska, P. Szperlich, D. Stróż, M. Sozańska, Opt. Mater. 35, 2208 (2013).

[3] C. Chen, L. Liu, Y. Lu, E. Siu-Wai Kong, Y. Zhang, X. Sheng, H. Ding, Carbon 45, 436 (2007).

[4] K. Łukasiewicz, A. Pietraszko, M. Kucharska, Ferroelectrics 375, 170 (2008).

[5] M. Nowak, P. Szperlich, Ł. Bober, J. Szala, G. Moskal, D. Stróż, Ultrason. Sonochem. 15, 709 (2008).

[6] M. Nowak, in: Nanowires Science and Technology, Ed. N. Lupu, Intech, Croatia 2010, p. 269. 